\title{
Stochastic Games for Verification of Probabilistic Timed Automata
}

\author{
Marta Kwiatkowska, Gethin Norman, and David Parker \\ Oxford University Computing Laboratory, Parks Road, Oxford, OX1 3QD
}

\begin{abstract}
Probabilistic timed automata (PTAs) are used for formal modelling and verification of systems with probabilistic, nondeterministic and real-time behaviour. For non-probabilistic timed automata, forwards reachability is the analysis method of choice, since it can be implemented extremely efficiently. However, for PTAs, such techniques are only able to compute upper bounds on maximum reachability probabilities. In this paper, we propose a new approach to the analysis of PTAs using abstraction and stochastic games. We show how efficient forwards reachability techniques can be extended to yield both lower and upper bounds on maximum (and minimum) reachability probabilities. We also present abstraction-refinement techniques that are guaranteed to improve the precision of these probability bounds, providing a fully automatic method for computing the exact values. We have implemented these techniques and applied them to a set of large case studies. We show that, in comparison to alternative approaches to verifying PTAs, such as backwards reachability and digital clocks, our techniques exhibit superior performance and scalability.
\end{abstract}

\section{Introduction}

Probabilistic behaviour occurs naturally in many real-time systems, either due to the use of randomisation, or because of the presence of unreliable components. Prominent examples include communication protocols such as Bluetooth, IEEE 802.11 and FireWire, which use randomised back-off schemes and are designed to function over faulty communication channels. Another important class are security protocols, such as for non-repudiation, anonymity and non-interference, where randomisation and timing are both essential ingredients.

Probabilistic timed automata (PTAs) 9116, which are finite state automata extended with real-valued clocks and discrete probabilistic choice, are a natural formalism for modelling and analysing such systems. Formal verification techniques for PTAs can help to identify anomalies resulting from the subtle interplay between probabilistic, real-time and nondeterministic aspects of these systems. A fundamental property of a PTA is the minimum or maximum probability of reaching a particular class of states in the model. This allows the expression of a wide range of useful properties, for example, "the minimum probability that a data packet is correctly delivered with $t$ seconds". 
There are three main existing algorithmic approaches to the verification of PTAs: (i) forwards reachability [16|5]; (ii) backwards reachability [17]; and (iii) digital clocks [15]. Forwards reachability is based on a symbolic forwards exploration, similar to the techniques implemented in state-of-the art tools for nonprobabilistic timed automata $6[18$. This approach is appealing because it can be implemented extremely efficiently with data structures such as difference-bound matrices (DBMs). However, in the context of probabilistic timed automata, these techniques yield only an upper bound on maximum reachability probabilities.

Backwards reachability [17] performs a state-space exploration in the opposite direction, from target to initial states. This computes exact values for both minimum and maximum reachability probabilities; however, the operations required to implement it are expensive, limiting its applicability. The digital clocks technique of [15] uses an efficient language-level translation to a probabilistic model with finite state semantics. This also gives precise values for minimum and maximum probabilities, but is only applicable to a restricted class of PTAs.

PTAs are, because of their real-valued model of time, inherently infinite-state. The three PTA verification techniques described above work by constructing a finite-state Markov decision process (MDP) that can be analysed with existing tools and techniques. This MDP can be viewed as an abstraction of the infinitestate semantics of the PTA. In this paper, we take a new approach, using the ideas of [13] to represent PTA abstractions as stochastic two-player games.

We first show how the forwards reachability technique of [16] can be generalised to produce a stochastic game that yields lower and upper bounds on either minimum or maximum reachability probabilities of PTAs. Then, using abstraction-refinement methods, we show how the stochastic game can be iteratively refined in order to tighten these bounds. This gives a fully automatic technique to compute exact reachability probabilities within a finite number of steps. Finally, we present a prototype tool implementing these techniques that exhibits significantly better performance than other PTA verification approaches. A full version of this paper, including proofs is also available [14.

Related work. Existing PTA verification techniques are discussed above and a detailed experimental comparison is included in Section 6. Also relevant is 3], which presents an algorithm for computing time-abstracting bisimulation quotients of PTAs. Abstraction-refinement approaches have been proposed for non-probabilistic timed automata, e.g. [12] which uses bounded model checking and SAT-based techniques, 21] which is based on the region graph construction, and [7] for verifying PLC automata using UPPAAL [18.

\section{Markov decision processes and stochastic games}

Markov decision processes (MDPs) are a widely used formalism for modelling systems that exhibit both nondeterministic and probabilistic behaviour.

Definition 1. An MDP M is a tuple $\left(S, \bar{S}\right.$, Act, Steps $\left._{\mathrm{M}}\right)$ where $S$ is a set of states, $\bar{S} \subseteq S$ is the set of initial states, Act is a set of actions and Steps $_{\mathrm{M}}$ : $S \times A c t \rightarrow \operatorname{Dist}(S)$ is the probabilistic transition function. 
In each state $s \in S$ of an MDP M, there is a nondeterministic choice between one or more available actions $a \in \operatorname{Act}$ (those for which $\operatorname{Steps}_{\mathrm{M}}(s, a)$ is defined). After the choice of an action $a$, a successor state is selected at random according to the probability distribution $\operatorname{Steps}_{\mathrm{M}}(s, a)$. A path through M is a sequence of states selected in this fashion.

To reason about the MDP M, we use the notion of an adversary, which is a possible resolution of all nondeterministic choices in $\mathrm{M}$ (formally, an adversary is a function from finite paths to actions). For a fixed adversary $A$, we can define a probability measure over the set of paths from a state $s$ and, in particular, the probability $p_{s}^{A}(F)$ of reaching a target $F \subseteq S$ from $s$ under $A$. We are typically interested in the minimum and maximum reachability probabilities for $F$ :

$$
p_{\mathrm{M}}^{\min }(F) \stackrel{\text { def }}{=} \inf _{s \in \bar{S}} \inf _{A} p_{s}^{A}(F) \text { and } p_{\mathrm{M}}^{\max }(F) \stackrel{\text { def }}{=} \sup _{s \in \bar{S}} \sup _{A} p_{s}^{A}(F) .
$$

These values, and an adversary of $M$ which produces them, can be computed with a simple numerical computation called value iteration [19].

Stochastic two-player games [20|4] extend MDPs by allowing two types of nondeterministic choice, controlled by separate players. We use stochastic games in the manner proposed in 13 to represent an abstraction of an MDP.

Definition 2. A stochastic game $\mathrm{G}$ is a tuple $\left(S, \bar{S}\right.$, Act $\left._{\text {Steps }} \mathrm{G}\right)$ where: $S$ is a set of states, $\bar{S} \subseteq S$ is the set of initial states Act is a set of actions and Steps $s_{\mathrm{G}}: S \times A c t \rightarrow 2^{\mathrm{Dist}(S)}$ is the probabilistic transition function.

Each transition of a stochastic game $\mathrm{G}$ comprises three choices: first, like for an MDP, player 1 picks an available action $a \in A c t$; next, player 2 selects a distribution $\lambda$ from the set $\operatorname{Steps}_{\mathrm{G}}(s, a)$; finally, a successor state is chosen at random according to $\lambda$. A resolution of the nondeterminism in $\mathrm{G}$ (the analogue of an MDP adversary) is a pair of strategies $\sigma_{1}, \sigma_{2}$ for the players, under which we can define the probability $p_{s}^{\sigma_{1}, \sigma_{2}}(F)$ of reaching a target $F \subseteq S$ from a state $s$.

Intuitively, the idea of [13] is that, in a stochastic game G, representing an abstraction of an MDP M, player 2 choices represent nondeterminism present in $\mathrm{M}$ and player 1 choices represent additional nondeterminism introduced through abstraction. By quantifying over strategies for players 1 and 2, we can obtain both lower bounds $(l b)$ and upper bounds $(u b)$ on the minimum and maximum reachability probabilities of $M$. If $G$ is constructed from $M$ using the approach of [13, then, in the case of maximum probabilities, for example:

$$
p_{\mathrm{G}}^{l b, \max }(F) \leqslant p_{\mathrm{M}}^{\max }(F) \leqslant p_{\mathrm{G}}^{u b, \max }(F)
$$

where, in the stochastic game G:

$$
\begin{gathered}
p_{\mathrm{G}}^{l b, \max }(F) \stackrel{\text { def }}{=} \sup _{s \in \bar{S}} \inf _{\sigma_{1}} \sup _{\sigma_{2}} p_{s}^{\sigma_{1}, \sigma_{2}}(F) \\
p_{\mathrm{G}}^{u b, \max }(F) \stackrel{\text { def }}{=} \sup _{s \in \bar{S}} \sup _{\sigma_{1}} \sup _{\sigma_{2}} p_{s}^{\sigma_{1}, \sigma_{2}}(F)
\end{gathered}
$$

Using similar techniques as those for MDPs, we can efficiently compute these values and strategies for players 1 and 2 that result in them [4]. 


\section{Probabilistic Timed Automata}

Time, clocks and zones. Probabilistic timed automata model time using clocks, variables over the set $\mathbb{R}$ of non-negative reals. We assume a finite set $\mathcal{X}$ of clocks. A function $v: \mathcal{X} \rightarrow \mathbb{R}$ is referred to as a clock valuation and the set of all clock valuations is denoted by $\mathbb{R}^{\mathcal{X}}$. For any $v \in \mathbb{R}^{\mathcal{X}}, t \in \mathbb{R}$ and $X \subseteq \mathcal{X}$, we use $v+t$ to denote the clock valuation which increments all clock values in $v$ by $t$ and $v[X:=0]$ for the valuation in which clocks in $X$ are reset to 0 .

The set of zones of $\mathcal{X}$, written Zones $(\mathcal{X})$, is defined by the syntax:

$$
\zeta::=\text { true }|x \leqslant d| c \leqslant x|x+c \leqslant y+d| \neg \zeta \mid \zeta \vee \zeta
$$

where $x, y \in \mathcal{X}$ and $c, d \in \mathbb{N}$. A zone $\zeta$ represents the set of clock valuations $v$ which satisfy $\zeta$, denoted $v \triangleleft \zeta$, i.e. those for which $\zeta$ resolves to true by substituting each clock $x$ with $v(x)$.

We will use several classical operations on zones $[8 \mid 22$. The zone $\nearrow \zeta$ contains all clock valuations that can be reached from a valuation in $\zeta$ by letting time pass. Conversely, $\measuredangle \zeta$ contains those that can reach $\zeta$ by letting time pass. For $X \subseteq \mathcal{X}$, the zone $[X:=0] \zeta$ contains the clock valuations which result in a valuation in $\zeta$ when the clocks in $X$ are reset to 0 , while $\zeta[X:=0]$ contains the valuations obtained from those in $\zeta$ by resetting these clocks to 0 .

Syntax and semantics of PTAs. We now present the formal syntax and semantics of probabilistic timed automata.

Definition 3. A PTA is a tuple $\mathrm{P}=(L, \bar{l}$, Act, inv, enab, prob) where:

- $L$ is a finite set of locations and $\bar{l} \in L$ is the initial location;

- Act is a finite set of actions;

- inv $: L \rightarrow \operatorname{Zones}(\mathcal{X})$ is the invariant condition;

- enab $: L \times A c t \rightarrow Z$ Zones $(\mathcal{X})$ is the enabling condition;

- prob : $L \times A c t \rightarrow \operatorname{Dist}\left(2^{\mathcal{X}} \times L\right)$ is the probabilistic transition function.

A state of a PTA is a pair $(l, v) \in L \times \mathbb{R}^{\mathcal{X}}$ such that $v \triangleleft i n v(l)$. In any state $(l, v)$, a certain amount of time $t \in \mathbb{R}$ can elapse, after which an action $a \in$ Act is performed. The choice of $t$ requires that, while time passes, the invariant inv $(l)$ remains continuously satisfied. Each action $a$ can be only chosen if it is enabled, that is, the zone $\operatorname{enab}(l, a)$ is satisfied by $v+t$. Once action $a$ is chosen, a set of clocks to reset and successor location are selected at random, according to the distribution $\operatorname{prob}(l, a)$. We call each element $\left(X, l^{\prime}\right) \in 2^{\mathcal{X}} \times L$ in the support of $\operatorname{prob}(l, a)$ an edge and, for convenience, assume that the set of such edges, denoted edges $(l, a)$, is an ordered list $\left\langle e_{1}, \ldots, e_{n}\right\rangle$.

Definition 4. Let $\mathrm{P}=(L, \bar{l}$, Act, inv, enab, prob) be a PTA. The semantics of $\mathrm{P}$ is defined as the (infinite-state) $M D P \llbracket \mathrm{P} \rrbracket=(S, \bar{S}, \mathbb{R} \times$ Act, Steps $\mathrm{P}$ ) where:

- $S=\left\{(l, v) \in L \times \mathbb{R}^{\mathcal{X}} \mid v \triangleleft i n v(l)\right\}$ and $\bar{S}=\{(\bar{l}, \mathbf{0})\}$;

- $\operatorname{Steps}_{\mathrm{P}}((l, v),(t, a))=\lambda$ if and only if $v+t^{\prime} \triangleleft$ inv $(l)$ for all $0 \leqslant t^{\prime} \leqslant t$, $v+t \triangleleft \operatorname{enab}(l, a)$ and, for any $\left(l^{\prime}, v^{\prime}\right) \in S$ :

$$
\lambda\left(l^{\prime}, v^{\prime}\right)=\sum\left\{\left|\operatorname{prob}(l, a)\left(X, l^{\prime}\right)\right| X \in 2^{\mathcal{X}} \wedge v^{\prime}=(v+t)[X:=0] \mid\right\} .
$$


Each transition of the semantics of the PTA is a time-action pair $(t, a)$, representing time passing for $t$ time units, followed by a discrete $a$-labelled transition. If Steps $_{\mathrm{P}}((l, v),(t, a))$ is defined and edges $(l, a)=\left\langle\left(l_{1}, X_{1}\right), \ldots,\left(l_{n}, X_{n}\right)\right\rangle$, we write $(l, v) \stackrel{t, a}{\longrightarrow}\left\langle s_{1}, \ldots, s_{n}\right\rangle$ where $s_{i}=\left(l_{i},(v+t)\left[X_{i}:=0\right]\right)$ for all $1 \leqslant i \leqslant n$.

We make several standard assumptions about probabilistic timed automata. Firstly, we restrict our attention to structurally non-Zeno automata [23. This class of models, which can be identified syntactically and in a compositional fashion [24], guarantees time-divergent behaviour. Secondly, for technical reasons, we assume all zones appearing in a PTA are diagonal-free [2].

Probabilistic Reachability. The minimum and maximum probabilities of reaching, from the initial state of a PTA $\mathrm{P}$, a certain target $F \subseteq L$ are:

$$
p_{\mathrm{P}}^{\min }(F)=p_{\llbracket \mathrm{P} \rrbracket}^{\min }\left(S_{F}\right) \quad \text { and } \quad p_{\mathrm{P}}^{\max }(F)=p_{\llbracket \mathrm{P} \rrbracket}^{\max }\left(S_{F}\right)
$$

where $S_{F}=\{(l, v) \mid v \triangleleft i n v(l) \wedge l \in F\}$. We can easily consider more expressive targets, that refer to both locations and clock values, through a simple syntactic modification of the PTA [16].

Symbolic states and operations. In order to represent sets of PTA states, we use the concept of a symbolic state: a pair $\mathbf{z}=(l, \zeta)$, comprising a location $l$ and a zone $\zeta$ over $\mathcal{X}$, representing the set of PTA states $\{(l, v) \mid v \triangleleft \zeta\}$. We use the notation $(l, v) \in(l, \zeta)$ to denote inclusion of a PTA state in a symbolic state.

We will use the time successor and discrete successor operations of [8]22]. For a symbolic state $(l, \zeta)$, action $a$, and edge $e=\left(X, l^{\prime}\right) \in \operatorname{edges}(l, a)$, we define:

$-\operatorname{tsuc}(l, \zeta) \stackrel{\text { def }}{=}(l, \operatorname{inv}(l) \wedge \nearrow \zeta)$ is the time successor of $(l, \zeta)$;

- dsuc $[a, e](l, \zeta) \stackrel{\text { def }}{=}\left(l^{\prime},(\zeta \wedge\right.$ enab $\left.(l, a))[X:=0] \wedge i n v\left(l^{\prime}\right)\right)$ is the discrete successor of $(l, \zeta)$ with respect to $e$;

$-\operatorname{post}[a, e](l, \zeta) \stackrel{\text { def }}{=} \operatorname{tsuc}(\operatorname{dsuc}[a, e](l, \zeta))$ is the post of $(l, \zeta)$ with respect to $e$.

The $c$-closure of a zone $\zeta$ is obtained by removing any constraint that refers to integers greater than $c$. For a given $c$, there are only a finite number of $c$-closed zones. For the remainder of this paper, we assume that all zones are $c$-closed where $c$ is the largest constant appearing in the PTA under study.

\section{Forwards Reachability for PTAs}

In this section, we begin by describing the approach of [16], which we will refer to as MDP-based forwards reachability. This computes only upper bounds on maximum reachability probabilities of a PTA. Subsequently, we will propose a new algorithm, based on stochastic games, which addresses these limitations.

\subsection{MDP-based forwards reachability}

The MDP-based forwards reachability approach of [16] works by building an abstraction of a PTA P. This abstraction is represented by an MDP M whose 
BuildReachGraph $(\mathrm{P}, F)$

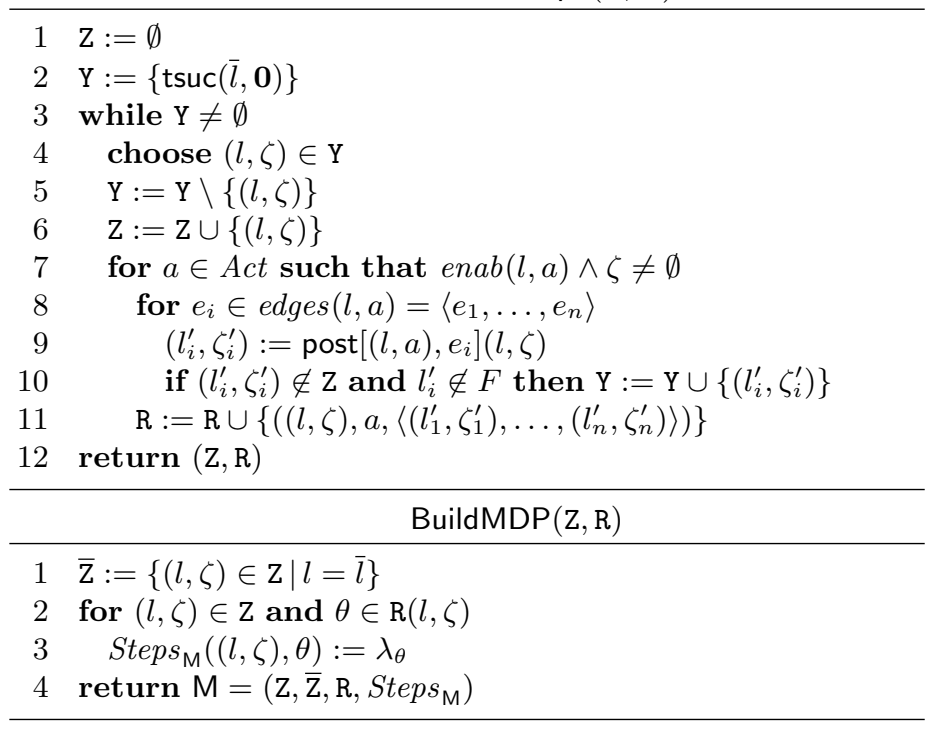

Fig. 1. Algorithm for MDP-based forwards reachability, based on [16]

state space is a set $\mathrm{Z}$ of symbolic states, i.e. each state of $\mathrm{M}$ represents a set of states of the infinite-state MDP semantics $\llbracket P \rrbracket$. The algorithm of [16] is shown in Figure 1. For the purposes of this presentation, we have reformulated the algorithm into: (i) the construction of a reachability graph over the set of symbolic states Z; and (ii) the construction of an MDP M from this graph.

The algorithm to build this reachability graph is based on the well-known forwards reachability algorithm for non-probabilistic timed automata 618]. It performs a forwards exploration through the automata, successively computing symbolic states using the post operation. One important difference is that, in the probabilistic setting, on-the-fly techniques cannot be used: the state-space exploration is exhaustive. This is because the aim is to determine, not just the existence of a path to the target, but the probability of reaching the target. For this, an MDP containing all such paths is constructed and analysed.

A reachability graph captures information about the transitions in a PTA. It comprises a multiset ${ }^{1} \mathrm{Z}$ of symbolic states and a set $\mathrm{R} \subseteq \mathrm{Z} \times A c t \times \mathrm{Z}^{+}$of symbolic transitions. Each symbolic transition $\theta \in \mathrm{R}$ takes the form:

$$
\theta=\left((l, \zeta), a,\left\langle\left(l_{1}, \zeta_{1}\right), \ldots,\left(l_{n}, \zeta_{n}\right)\right\rangle\right)
$$

where $n=|\operatorname{edges}(l, a)|$. Intuitively, $\theta$ represents the possibility of taking action $a$ from a PTA state in $(l, \zeta)$ and, for each edge $\left(X_{i}, l_{i}\right) \in$ edges $(l, a)$, reaching a state in $\left(l_{i}, \zeta_{i}\right)$. A key property of symbolic transitions is the notion of validity:

$$
\operatorname{valid}(\theta) \stackrel{\text { def }}{=} \zeta \wedge \swarrow\left(\operatorname{enab}(l, a) \wedge\left(\wedge_{i=1}^{n}\left(\left[X_{i}:=0\right] \zeta_{i}\right)\right)\right)
$$

\footnotetext{
1 The use of a multiset is a technical requirement, later used for abstraction refinement.
} 
which gives precisely the set of clock valuations satisfying $\zeta$ from which it is possible to let time pass and perform the action $a$ such that taking the $i$ th edge $\left(X_{i}, l_{i}\right)$ gives a state in $\left(l_{i}, \zeta_{i}\right)$. A symbolic transition $\theta$ is valid if the zone $\operatorname{valid}(\theta)$ is non-empty. This leads to the following formal definition of a reachability graph.

Definition 5. A reachability graph for a PTA $\mathrm{P}=(L, \bar{l}, A c t$, inv, enab, prob $)$ and target $F$, is a pair $(\mathrm{Z}, \mathrm{R})$ where:

$-\mathrm{Z} \subseteq L \times \operatorname{Zones}(\mathcal{X})$ is a multiset of symbolic states where $\{s \in \mathbf{z} \mid \mathbf{z} \in \mathrm{Z}\}=S$;

$-\mathrm{R} \subseteq \mathrm{Z} \times A$ ct $\times \mathrm{Z}^{+}$is a set of valid symbolic transitions;

and, if $\mathrm{z}=(l, \zeta) \in \mathrm{Z}, l \notin F, s \in \mathrm{z}$ and $s \stackrel{t, a}{\longrightarrow}\left\langle s_{1}, \ldots, s_{n}\right\rangle$, then $\mathrm{R}$ contains $a$ symbolic transition $\left(\mathbf{z}, a,\left\langle\mathbf{z}_{1}, \ldots, \mathbf{z}_{n}\right\rangle\right)$ such that $s_{i} \in \mathbf{z}_{i}$ for all $1 \leqslant i \leqslant n$.

For any PTA $\mathrm{P}$ and target $F$, it follows from the definition of post that algorithm BuildReachGraph $(\mathrm{P}, F)$ in Figure 1 returns a (unique) reachability graph for $(\mathrm{P}, F)$. However, the above conditions do not imply the uniqueness of reachability graphs, and there may exist many other such graphs for $(\mathrm{P}, F)$.

Given a reachability graph $(Z, R)$ we can construct an MDP $M$ with state space $\mathrm{Z}$ using the symbolic transitions in $\mathrm{R}$ to build the transitions of $\mathrm{M}$. More precisely, a symbolic transition $\theta=\left((l, \zeta), a,\left\langle\left(l_{1}, \zeta_{1}\right), \ldots,\left(l_{n}, \zeta_{n}\right)\right\rangle\right)$ induces a probability distribution $\lambda_{\theta}$ over symbolic states $\mathrm{Z}$ where for any $\left(l^{\prime}, \zeta^{\prime}\right) \in \mathrm{Z}$ :

$$
\lambda_{\theta}\left(l^{\prime}, \zeta^{\prime}\right) \stackrel{\text { def }}{=} \sum\left\{\left|\operatorname{prob}(l, a)\left(e_{i}\right)\right| e_{i} \in \operatorname{edges}(l, a) \wedge \zeta_{i}=\zeta^{\prime} \mid\right\} .
$$

Using these distributions, the algorithm $\operatorname{BuildMDP}(Z, R)$ in Figure 1 constructs an MDP M, analysis of which yields bounds on the behaviour of $P$.

Theorem 1. Let $\mathrm{P}$ be a PTA with target $F$. If $(\mathrm{Z}, \mathrm{R})$ is a reachability graph for $(\mathrm{P}, F)$ and $\mathrm{M}$ is the $M D P$ returned by $\operatorname{BuildMDP}(\mathrm{Z}, \mathrm{R})$ (see Figure 1), then $p_{\mathrm{M}}^{\min }\left(\mathrm{Z}_{F}\right) \leqslant p_{\mathrm{P}}^{\min }(F)$ and $p_{\mathrm{P}}^{\max }(F) \leqslant p_{\mathrm{M}}^{\max }\left(\mathrm{Z}_{F}\right)$ where $\mathrm{Z}_{F}=F \times$ Zones $(\mathcal{X})$.

This theorem extends [16], by establishing the result for any reachability graph, not just that returned by BuildReachGraph and, by restricting to structurally non-Zeno PTAs, also yields lower bounds on minimum reachability probabilities.

Example 1. We illustrate these ideas using the simple PTA P in Figure 2(a). We use the standard graphical notation for PTAs and omit probability 1 labels. Applying BuildReachGraph $\left(\mathrm{P},\left\{l_{3}\right\}\right)$ (see Figure 1) yields the symbolic states:

$$
\mathrm{Z}=\left\{\left(l_{0}, x=y\right),\left(l_{1}, x=y\right),\left(l_{1}, y<x-2\right),\left(l_{2}, x \leqslant y\right),\left(l_{3}, x=y\right)\right\}
$$

and the set of symbolic transitions R. From the first two symbolic states, for example, we have $\mathrm{R}\left(l_{0}, x=y\right)=\left\{\theta_{a}\right\}$ and $\mathrm{R}\left(l_{1}, x=y\right)=\left\{\theta_{b}, \theta_{c}\right\}$ where:

$$
\begin{aligned}
\theta_{a} & =\left(\left(l_{0}, x=y\right), a,\left\langle\left(l_{1}, x=y\right),\left(l_{2}, x \leqslant y\right)\right\rangle\right) \\
\theta_{b} & =\left(\left(l_{1}, x=y\right), b,\left\langle\left(l_{1}, x=y\right)\right\rangle\right), \theta_{c}=\left(\left(l_{1}, x=y\right), c,\left\langle\left(l_{3}, x=y\right)\right\rangle\right)
\end{aligned}
$$




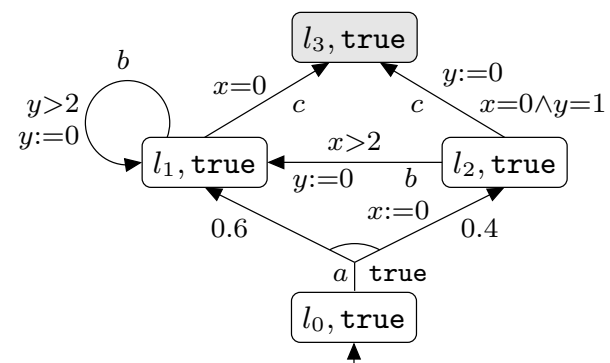

(a) PTA

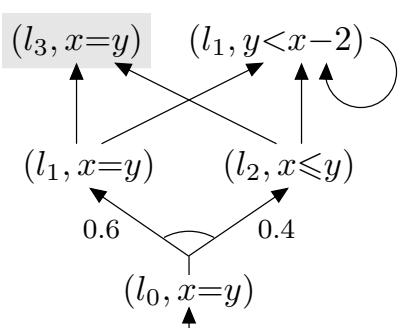

(b) MDP

Fig. 2. Analysis of a PTA through MDP-based forwards reachability

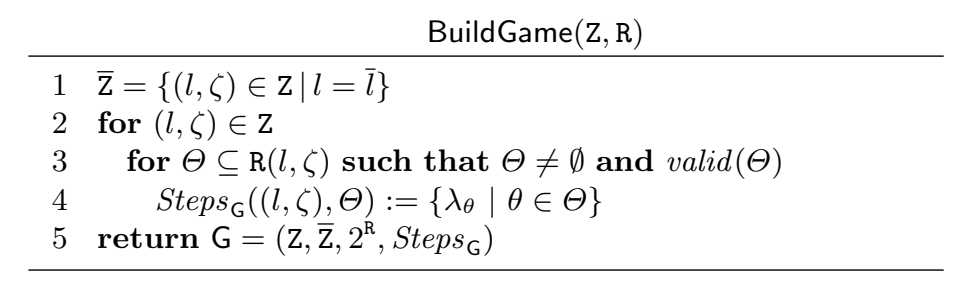

Fig. 3. Algorithm to construct a stochastic game from a reachability graph

The resulting MDP is shown in Figure 2(b). The maximum probability of reaching location $l_{3}$ in the PTA is 0.6 , which results from taking action $a$ in $l_{0}$ immediately and, if $l_{1}$ is reached, proceeding straight to $l_{3}$. An alternative is to wait for 1 time unit in $l_{0}$ and then take $a$, reaching $l_{3}$ via $l_{2}$, however, this results in a lower probability of 0.4 . An upper bound on the maximum probability for the PTA is obtained from the maximum probability of reaching $\left(l_{3}, x=y\right)$ in the MDP. The resulting value is 1 . This is because the symbolic states for locations $l_{1}$ and $l_{2}$ are too coarse to preserve the precise time that action $a$ is taken.

\subsection{Game-based forwards reachability}

The main limitation of the MDP-based forwards reachability algorithm is that it only provides lower bounds for minimum and upper bounds for maximum reachability probabilities. We now describe how to construct, from a reachability graph, a stochastic game $\mathrm{G}$ that yields both lower and upper bounds. The game $\mathrm{G}$ is, like the MDP in the previous section, an abstraction of the infinite-state MDP semantics of the PTA, whose state space is the symbolic states Z.

We utilise the approach of [13] to represent an abstraction of an MDP as a stochastic two-player game. The basic idea is that the two players in the game represent nondeterminism introduced by the abstraction and nondeterminism from the original model. In a symbolic state $(l, \zeta)$ of the game abstraction of a PTA, player 1 first picks a PTA state $(l, v) \in(l, \zeta)$ and then player 2 makes a choice over the actions that become enabled after letting time pass from $(l, v)$.

In order to construct such a game from a reachability graph $(Z, R)$, we first extend the notion of validity to sets of symbolic transitions with the same source. 


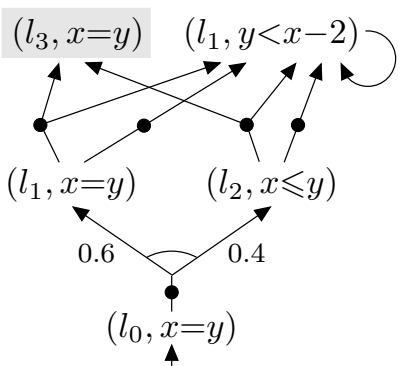

(a) From reachability graph

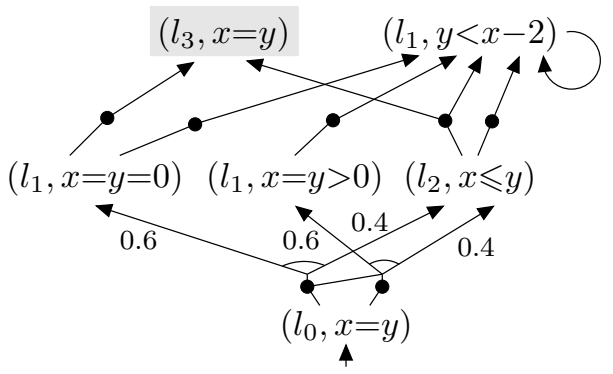

(b) After one refinement

Fig. 4. Stochastic games for the PTA example of Figure2

For any symbolic state $(l, \zeta) \in \mathrm{Z}$ and set of symbolic transitions $\Theta \subseteq \mathrm{R}(l, \zeta)$, let:

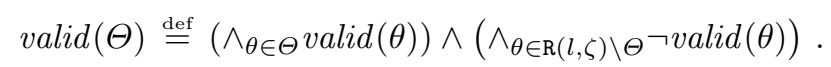

By construction, valid $(\Theta)$ identifies precisely the clock valuations $v \triangleleft \zeta$ such that, from $(l, v)$, it is possible to perform a transition encoded by any symbolic transition $\theta \in \Theta$, but it is not possible to perform a transition encoded by any other symbolic transition of $\mathrm{R}(l, \zeta)$.

The algorithm BuildGame in Figure 3 describes how to construct, from a reachability graph $R$, a stochastic game with symbolic states $Z$. In a state $z$ of the game, player 1 chooses between any non-empty valid set of symbolic transitions $\Theta \subseteq R(z)$. Player 2 then selects a symbolic transition $\theta \in \Theta$. As the following result demonstrates, this game yields lower and upper bounds on either minimum or maximum reachability probabilities of the PTA.

Theorem 2. Let $\mathrm{P}$ be a PTA with target $F$. If $(\mathrm{Z}, \mathrm{R})$ is a reachability graph for

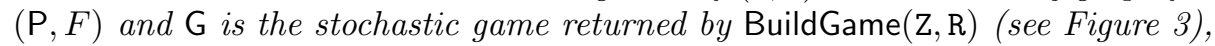
then $p_{\mathrm{G}}^{l b, \star}\left(\mathrm{Z}_{F}\right) \leqslant p_{\mathrm{P}}^{\star}(F) \leqslant p_{\mathrm{G}}^{u b, \star}\left(\mathrm{Z}_{F}\right)$ for $\star \in\{\min , \max \}$.

Example 2. We return to the PTA from Figure 2 and the reachability graph constructed in Example 1. The corresponding stochastic game is shown in Figure 4(a) As for PTAs and MDPs, we draw probability distributions as arrows grouped by an arc, omitting the labelling of probability 1 transitions. A set of distributions emanating from a black circle indicates a player 2 choice; the outgoing edges from each symbolic state represent a player 1 choice.

Consider, the symbolic state $\left(l_{1}, x=y\right)$, for which there are two symbolic transitions $\theta_{b}$ and $\theta_{c}$ (see Example1). Since $\operatorname{valid}\left(\theta_{b}\right)=(x=y)$ and $\operatorname{valid}\left(\theta_{c}\right)=(x=y=0)$, we have $\operatorname{valid}\left(\left\{\theta_{b}\right\}\right)=(x=y>0), \operatorname{valid}\left(\left\{\theta_{c}\right\}\right)=\emptyset$ and $\operatorname{valid}\left(\left\{\theta_{b}, \theta_{c}\right\}\right)=(x=y=0)$. This tells us that there are two classes of PTA states in $\left(l_{1}, x=y\right)$ : those in which both actions $b$ and $c$ become enabled, and those in which only $b$ becomes enabled. Thus, in the game state (see Figure 4(a) , we see that player 1 chooses between these two classes and then player 2 chooses an available action.

Using Theorem 2, the stochastic game in Figure 4(a) gives bounds on the maximum probability of reaching $l_{3}$ in the PTA. The upper bound (as for the 
Refine $\left(\mathrm{Z}, \mathrm{R},(l, \zeta), \Theta_{l b}, \Theta_{u b}\right)$

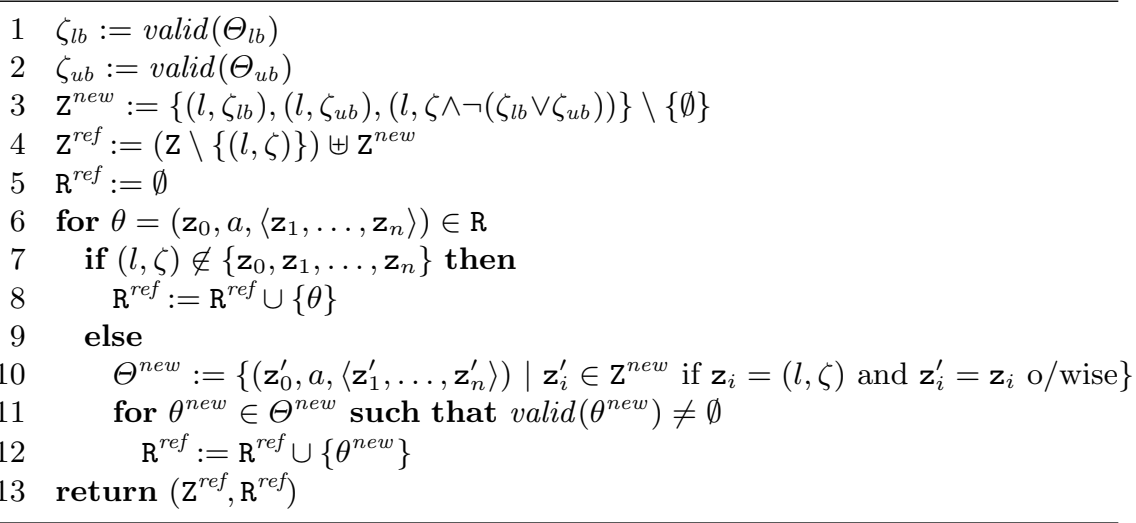

Fig. 5. Algorithm to refine symbolic state $(l, \zeta)$ in reachability graph $(\mathrm{Z}, \mathrm{R})$

MDP) is 1 as, after either branch of the initial probabilistic choice, player 1 can make a choice which allows $l_{3}$ to be reached with probability 1 . The lower bound, however, is 0 because player 1 can also, in both cases, make $l_{3}$ unreachable.

As the above example illustrates, it is possible that the difference between the lower and upper bounds from the game is too great to provide useful information. In the next section, we will address this issue by introducing a way to refine the abstraction to reduce the difference between the bounds.

\section{Abstraction Refinement}

The game-based abstraction approach of [13] has been extended with refinement techniques in [1011]. Inspired by non-probabilistic counterexample-guided abstraction refinement, the idea is that an initially coarse abstraction is iteratively refined until it is precise enough to yield useful verification results. Crucial to this approach is the use of the lower and upper bounds provided by a stochastic game abstraction as a quantitative measure of the preciseness of the abstraction.

The refinement algorithm. Our refinement algorithm takes a reachability graph $(Z, R)$, splits one or more of the symbolic states in $\mathrm{Z}$ and then modifies the symbolic transitions of $R$ accordingly. This process is guided by the analysis of the stochastic game constructed from $(\mathrm{Z}, \mathrm{R})$, i.e. the bounds for the probability of reaching the target and player 1 strategies that attain these bounds.

We now outline the refinement of a single symbolic state $(l, \zeta)$ for which the bounds differ and for which distinct player 1 strategies yield each bound 2 A player 1 strategy chooses, for any state in the stochastic game, an action available in the state. By construction, an available action in $(l, \zeta)$ is a valid set

\footnotetext{
${ }^{2}$ From the results of [13] such a state exists when the bounds differ in some state.
} 




Fig. 6. Abstraction-refinement loop to compute reachability probabilities

of symbolic transitions from $\mathrm{R}(l, \zeta)$. We let $\Theta_{l b}, \Theta_{u b} \subseteq \mathrm{R}(l, \zeta)$ denote the distinct player 1 strategy choices for the lower and upper bound respectively. Since the validity conditions for $\Theta_{l b}$ and $\Theta_{u b}$ identify precisely the clock valuations in $\zeta$ for which the corresponding transitions of $\llbracket \mathrm{P} \rrbracket$ are possible, we split $(l, \zeta)$ into:

$$
\left(l, \operatorname{valid}\left(\Theta_{l b}\right)\right), \quad\left(l, \operatorname{valid}\left(\Theta_{u b}\right)\right) \text { and }\left(l, \zeta \wedge \neg\left(\operatorname{valid}\left(\Theta_{l b}\right) \vee \operatorname{valid}\left(\Theta_{u b}\right)\right)\right) .
$$

By construction, $\operatorname{valid}\left(\Theta_{l b}\right)$ and $\operatorname{valid}\left(\Theta_{u b}\right)$ are both non-empty. Furthermore, since $\Theta_{l b} \neq \Theta_{u b}$, from the definition of validity, we have $\operatorname{valid}(\Theta) \wedge \operatorname{valid}\left(\Theta^{\prime}\right)=\emptyset$, and hence the split of $(l, \zeta)$ produces a strict refinement of $\mathrm{Z}$.

The complete refinement algorithm is shown in Figure 5. Lines 1-4 refine $\mathbf{Z}$, as just described, and lines 5-12 update the set of symbolic transitions R. The result is a new reachability graph, for which the corresponding stochastic game is a refined abstraction of the PTA, satisfying the following properties.

Theorem 3. Let $\mathrm{P}$ be a PTA with target $F$ and $(\mathrm{Z}, \mathrm{R})$ be a reachability graph for $(\mathrm{P}, F)$. If $\left(\mathrm{Z}^{\text {ref }}, \mathrm{R}^{\text {ref }}\right)$ is the result of applying algorithm Refine (see Figure 5) to $(\mathrm{Z}, \mathrm{R}), \mathrm{G}=$ BuildGame $(\mathrm{Z}, \mathrm{R})$ and $\mathrm{G}^{\text {ref }}=\operatorname{BuildGame}\left(\mathrm{Z}^{\text {ref }}, \mathrm{R}^{\text {ref }}\right)$, then:

(i) $\left(\mathrm{Z}^{\text {ref }}, \mathrm{R}^{\text {ref }}\right)$ is a reachability graph for $(\mathrm{P}, F)$;

(ii) $p_{\mathrm{G}}^{l b, \star}\left(\mathrm{Z}_{F}\right) \leqslant p_{\mathrm{G}^{\text {ref }}}^{l b, \star}\left(\mathrm{Z}_{F}\right)$ and $p_{\mathrm{G}^{\text {ref }}}^{u b, \star}\left(\mathrm{Z}_{F}\right) \leqslant p_{\mathrm{G}}^{u b, \star}\left(\mathrm{Z}_{F}\right)$ for $\star \in\{\min , \max \}$.

This refinement scheme, applied in a iterative manner, provides a way of computing exact values for minimum or maximum reachability probabilities of a PTA. This algorithm, outlined in Figure 6, starts with the reachability graph constructed through forwards reachability and then repeatedly: (i) builds a stochastic game; (ii) solves the game to obtain lower and upper bounds; and (iii) refines the reachability graph, based on an analysis of the game. The iterative process terminates when the difference between the bounds falls below a given level of precision $\varepsilon$. In fact, as the following result states, this process is guaranteed to terminate, in a finite number of steps, with the precise answer.

Theorem 4. Let $\mathrm{P}$ be a PTA with target $F$ and $\star \in\{\min , \max \}$. The algorithm AbstractRefine $(\mathrm{P}, F, \star, 0)$ (see Figure 6) terminates after a finite number of steps and returns $\left[p_{\mathrm{G}}^{l b, \star}, p_{\mathrm{G}}^{u b, \star}\right]$ where $p_{\mathrm{G}}^{l b, \star}=p_{\mathrm{P}}^{\star}(F)=p_{\mathrm{G}}^{u b, \star}$. 
Example 3. We return to our running example (see Figures 2 and 4 ) and consider the refinement of $\left(l_{1}, x=y\right)$, from which the lower and upper bounds on the maximum probability of reaching location $l_{3}$ are 0 and 1 . The player 1 strategies (see Example 2) to achieve these bounds select $\Theta_{l b}=\left\{\theta_{b}\right\}$ and $\Theta_{u b}=\left\{\theta_{b}, \theta_{c}\right\}$, respectively. The validity conditions for these choices are $(x=y>0)$ and $(x=y=0)$, and hence $\left(l_{1}, x=y\right)$ is divided into $\mathbf{z}_{1}=\left(l_{1}, x=y>0\right)$ and $\mathbf{z}_{2}=\left(l_{1}, x=y\right)$.

We then update the set $\mathrm{R}$, as described in Figure 5, splitting symbolic transitions whose source or target is $\left(l_{1}, x=y\right)$. For example, $\theta_{a}, \theta_{b}$ and $\theta_{c}$ (see Example 1) are split into, for $i=1,2$ :

$$
\theta_{a}^{i}=\left(\left(l_{0}, x=y\right), a,\left\langle\mathbf{z}_{i},\left(l_{2}, x \leqslant y\right)\right\rangle\right), \theta_{b}^{i}=\left(\mathbf{z}_{i}, b,\left\langle\mathbf{z}_{i}\right\rangle\right) \text { and } \theta_{c}^{i}=\left(\mathbf{z}_{i}, c,\left\langle\left(l_{3}, x=y=0\right)\right\rangle\right) .
$$

After removing $\theta_{c}^{2}$, which is not valid, the resulting stochastic game is shown in Figure 4(b). While this still yields bounds of $[0,1]$ for the initial state, two subsequent refinement tighten this to $[0.6,1.0]$ and then $[0.6,0.6]$.

\section{Experimental Results}

Implementation. We have implemented a prototype PTA model checker based on the techniques in this paper. It uses difference-bound matrices (DBMs) to represent zones. Since refinement can introduce non-convex zones, we also employ lists of DBMs. Our tool takes a textual description of a PTA (or the parallel composition of several PTAs) and a set of target locations. It then executes the abstraction-refinement loop described in Section 5 to compute either the minimum or maximum reachability probability.

Several aspects of the abstraction-refinement implementation merit further discussion. In particular, the refinement process presented in Section 5 discusses the refinement of a single symbolic state. Because each refinement requires a potentially expensive numerical solution phase, an efficient scheme to select which state (or states) are to be split is essential. In fact, we found it possible to obtain very good performance with relatively simple heuristics. In the results presented here, we simply refine all states for which the lower and upper bounds differ.

Our implementation includes several useful optimisations. Firstly, we modify the BuildGame algorithm so that it only rebuilds states of a stochastic game that have actually been modified during refinement. Secondly, we use the techniques described in 10, to re-use numerical results between refinement iterations, reducing the amount of numerical solution required.

Experimental results. We evaluate our implementation on 7 large PTA case studies from the literature: (i) csma and csma abst, two models of the IEEE 802.3 CSMA/CD protocol; (ii) firewire and firewire abst, two models of the IEEE 1394 FireWire root contention protocol; (iii) zeroconf, the Zeroconf network configuration protocol; and (iv) nrp honest and nrp malicious, two model of Markowitch \& Roggeman's non-repudiation protocol. Full details of all these case studies, their parameters, and the properties checked are available $3^{3}$

\footnotetext{
${ }^{3}$ http://www.prismmodelchecker.org/files/formats09/
} 


\begin{tabular}{|c|c|c|c|c|c|c|c|c|c|}
\hline \multirow{2}{*}{\multicolumn{2}{|c|}{$\begin{array}{c}\text { Case study } \\
\text { (parameters) } \\
{[\mathrm{min} / \mathrm{max}]}\end{array}$}} & \multicolumn{3}{|c|}{$\begin{array}{l}\text { Game-based } \\
\text { verification }\end{array}$} & \multicolumn{2}{|c|}{$\begin{array}{c}\text { Backwards } \\
\text { reachability } 17\end{array}$} & \multicolumn{2}{|c|}{$\begin{array}{c}\text { Digital clocks } \\
15\end{array}$} & \multirow{2}{*}{$\begin{array}{l}\text { Min/Max } \\
\text { reachability } \\
\text { probability }\end{array}$} \\
\hline & & Iters & States & Time (s) & States & Time (s) & States & Time (s) & \\
\hline \multirow{4}{*}{$\begin{array}{c}\text { csma } \\
\text { (max_backoff } \\
\text { collisions) } \\
{[\max ]} \\
\end{array}$} & 24 & 10 & 6,476 & 3.9 & 243 & 20.7 & $\mathrm{n} / \mathrm{a}$ & $\mathrm{n} / \mathrm{a}$ & 0.143555 \\
\hline & 28 & 10 & 18,196 & 8.9 & 575 & 77.8 & $\mathrm{n} / \mathrm{a}$ & $\mathrm{n} / \mathrm{a}$ & 0.005259 \\
\hline & 44 & 10 & 34,826 & 20.5 & 303 & 1443.7 & $\mathrm{n} / \mathrm{a}$ & $\mathrm{n} / \mathrm{a}$ & 0.076904 \\
\hline & 48 & 10 & 239,298 & 431.4 & time out & time out & $\mathrm{n} / \mathrm{a}$ & $\mathrm{n} / \mathrm{a}$ & $1.65 \mathrm{e}-5$ \\
\hline \multirow{4}{*}{$\begin{array}{c}\text { csma } \\
\text { abst } \\
\text { (deadline) } \\
\text { [min] }\end{array}$} & $\infty$ & 0 & 117 & 0.2 & 0 & 8.7 & 5240 & 21.2 & 1.0 \\
\hline & 1000 & 0 & 6,392 & 1.9 & 366 & 68.2 & $1,876,105$ & 71.2 & 0.0 \\
\hline & 2000 & 37 & 24,173 & 20.7 & 722 & 367.8 & $6,570,692$ & 651.8 & 0.86 \\
\hline & 3000 & 76 & 79,608 & 448.0 & 1,736 & 1436.3 & $11,780,692$ & 1951.9 & 0.99 \\
\hline \multirow{4}{*}{$\begin{array}{c}\text { firewire } \\
\text { (deadline) } \\
{[\text { min }]}\end{array}$} & $\infty$ & 0 & 257 & 0.7 & 127 & 26.4 & 212,268 & 39.7 & 1.0 \\
\hline & 25 & 0 & 1,369 & 2.0 & 1,004 & 839.5 & $14,089,691$ & 324.6 & 0.5 \\
\hline & 50 & 17 & 4,215 & 10.6 & 3,096 & 3149.9 & time out & time out & 0.78125 \\
\hline & 75 & 34 & 10,252 & 83.4 & time out & time out & mem out & mem out & 0.931641 \\
\hline \multirow{4}{*}{$\begin{array}{c}\text { firewire } \\
\text { abst } \\
\text { (deadline) } \\
\text { [min] }\end{array}$} & $\infty$ & 0 & 10 & 0.03 & 0 & 1.0 & 776 & 0.3 & 1.0 \\
\hline & 50 & 7 & 205 & 0.25 & 63 & 2.4 & 298,010 & 14.5 & 25 \\
\hline & 100 & 19 & 1,023 & 1.76 & 180 & 3.8 & 686,008 & 36.4 & 731 \\
\hline & 200 & 40 & 9,059 & 26.1 & 640 & 26.4 & $1,462,010$ & 149.2 & 0.999630 \\
\hline \multirow{4}{*}{$\begin{array}{c}\text { zeroconf } \\
\text { (deadline) } \\
{[\max ]}\end{array}$} & $\infty$ & 0 & 26 & 0.17 & 19 & 0.22 & 357 & 1.69 & 0.001302 \\
\hline & 100 & 0 & 132 & 0.16 & 15 & 0.32 & 8,423 & 0.93 & $6.52 \mathrm{e}-4$ \\
\hline & 150 & 13 & 380 & 0.44 & 101 & 0.72 & 23,888 & 1.71 & 0.001073 \\
\hline & 200 & 17 & 670 & 0.73 & 274 & 4.77 & 41,713 & 2.92 & 0.001222 \\
\hline \multirow{4}{*}{$\begin{array}{c}\text { nrp } \\
\text { honest } \\
\text { (deadline) } \\
\text { [min] }\end{array}$} & $\infty$ & 0 & 5 & 0.04 & 0 & 0.70 & $\mathrm{n} / \mathrm{a}$ & $\mathrm{n} / \mathrm{a}$ & 1.0 \\
\hline & 40 & 19 & 428 & 1.80 & 33 & 5.25 & $\mathrm{n} / \mathrm{a}$ & $\mathrm{n} / \mathrm{a}$ & 580 \\
\hline & 80 & 39 & 1,448 & 3.56 & 63 & 6.18 & $\mathrm{n} / \mathrm{a}$ & $\mathrm{n} / \mathrm{a}$ & 915 \\
\hline & 100 & 49 & 2,183 & 5.35 & 78 & 6.97 & $\mathrm{n} / \mathrm{a}$ & $\mathrm{n} / \mathrm{a}$ & 0.920234 \\
\hline \multirow{4}{*}{$\begin{array}{c}n r p \\
\text { malicious } \\
\text { (deadline) } \\
{[\mathrm{max}]}\end{array}$} & $\infty$ & 11 & 351 & 1.3 & 62 & 1.5 & $\mathrm{n} / \mathrm{a}$ & $\mathrm{n} / \mathrm{a}$ & 0.105658 \\
\hline & 5 & 3 & 1,663 & 1.5 & 75 & 2.9 & $\mathrm{n} / \mathrm{a}$ & $\mathrm{n} / \mathrm{a}$ & 0.1 \\
\hline & 10 & 15 & 8,080 & 11.1 & 408 & 117.3 & $\mathrm{n} / \mathrm{a}$ & $\mathrm{n} / \mathrm{a}$ & 0.105444 \\
\hline & 20 & 7 & 49,622 & 218.1 & 1,108 & 1606.5 & $\mathrm{n} / \mathrm{a}$ & $\mathrm{n} / \mathrm{a}$ & 0.105657 \\
\hline
\end{tabular}

Table 1. Performance statistics and comparisons for game-based PTA verification

We present a comparison of our implementation with the two other existing techniques for reachability analysis of PTAs: backwards reachability [17] and digital clocks [15. For the former, we use the implementation of [17] which uses PRISM as a back-end to analyse MDP. For the latter, we use a simple languagelevel translation. We do not consider the MDP-based forwards reachability algorithm [16 5] since this does compute exact probability values and is thus not directly comparable. All experiments were run on a $2 \mathrm{GHz}$ PC with 2GB RAM. Any run exceeding a time-limit of 1 hour was disregarded.

Table 1 summarises the experimental results. We give, for each PTA and each applicable analysis technique ${ }^{4}$ the total time required and the size of the probabilistic model constructed. For backwards reachability and digital clocks, this model is an MDP; for our approach, it is a stochastic game (we give the size of the final game constructed during abstraction-refinement). For backwards reachability, the time given includes both generation of an MDP and its solution in PRISM; for digital clocks, the value is just the solution time in PRISM. For our game-based verification approach, we give the total time for all steps: reachability graph generation and multiple iterations of game construction, solution and analysis. The number of refinement steps required is also shown; in all cases, we refine until precise values are obtained (i.e. $\varepsilon=0$ ). Finally, Table 1 also gives

\footnotetext{
${ }^{4}$ The digital clocks approach is not applicable to several of the case studies since the PTAs contain zones with strict constraints.
} 
the actual reachability probability for each model checking query and whether this a minimum or maximum value.

Analysis of the results. Our game-based approach to PTA verification performs extremely well. In all cases, it is faster than both backwards reachability and digital clocks, often by several orders of magnitude. We are also able to analyse PTAs too large to be verified using the digital clocks approach.

In terms of the size of the probabilistic models generated by the three techniques, we find that backwards reachability usually yields the smallest state spaces. This is because it only considers symbolic states for which the required probability is greater than 0 . Thanks to the fact that our approach avoids some of the complex zone operations required for backwards reachability, we are able to consistently outperform it, despite this fact. On PTAs with a very small number of clocks (e.g. firewire abst has only 2), the overhead of these complex operations is reduced and backwards reachability performs better. By contrast, for PTAs with more clocks (firewire has 7 and csma has 5), the opposite is true.

The reason that our game-based technique outperforms the digital clocks approach is that the latter generates models with much larger state spaces, which are slow to analyse, even with the efficient symbolic techniques of PRISM.

\section{Conclusions}

We have presented a novel technique for the verification of probabilistic automata, based on the use of two-player stochastic games to represent abstractions of their semantics. Our approach generates lower and upper bounds for either minimum or maximum reachability probabilities and then iteratively refines the game to compute the exact values in a finite number of steps. We have implemented this process and shown that it outperforms existing PTA verification techniques on a wide range of large case studies.

Our approach can easily be extended to compute expected-reward properties for the case where rewards are associated with transitions of a PTA. Furthermore, we plan to adapt our techniques to compute lower and upper bounds on more general classes of rewards properties. Another direction of future work is the investigation of improved abstraction-refinement schemes. The simple approach adopted in this paper works very well but we anticipate that there is considerable scope for improving performance further in this way. Finally, we also plan to apply this approach to the verification of real-time properties of software.

Acknowledgments. The authors are supported in part by EPSRC grants EP/D07956X and EP/D076625.

\section{References}

1. D. Beauquier. Probabilistic timed automata. Theoretical Computer Science, 292(1):65-84, 2003.

2. P. Bouyer. Untameable timed automata! In Proc. STACS'03, volume 2607 of LNCS, pages 620-631. Springer, 2003. 
3. T. Chen, T. Han, and J.-P. Katoen. Time-abstracting bisimulation for probabilistic timed automata. In Proc. TASE'08, pages 177-184. IEEE CS Press, 2008.

4. A. Condon. The complexity of stochastic games. Inf. and Comp., 96(2):203-224, 1992.

5. C. Daws, M. Kwiatkowska, and G. Norman. Automatic verification of the IEEE 1394 root contention protocol with KRONOS and PRISM. International Journal on Software Tools for Technology Transfer (STTT), 5(2-3):221-236, 2004.

6. C. Daws, A. Olivero, S. Tripakis, and S. Yovine. The tool Kronos. In Hybrid Systems III, volume 1066 of LNCS, pages 208-219. Springer, 1996.

7. H. Dierks, S. Kupferschmid, and K. Larsen. Automatic abstraction refinement for timed automata. In Proc. FORMATS'O', volume 4763 of LNCS, pages 114-129. Springer, 2007.

8. T. Henzinger, X. Nicollin, J. Sifakis, and S. Yovine. Symbolic model checking for real-time systems. Inf. and Comp., 111(2):193-244, 1994.

9. H. Jensen. Model checking probabilistic real time systems. In Proc. 7th Nordic Workshop on Programming Theory, pages 247-261, 1996.

10. M. Kattenbelt, M. Kwiatkowska, G. Norman, and D. Parker. A game-based abstraction-refinement framework for Markov decision processes. Technical Report RR-08-06, Oxford University Computing Laboratory, February 2008.

11. M. Kattenbelt, M. Kwiatkowska, G. Norman, and D. Parker. Abstraction refinement for probabilistic software. In Proc. VMCAI'09, volume 5403 of LNCS, pages 182-197. Springer, 2009.

12. S. Kemper and A. Platzer. SAT-based abstraction refinement for real-time systems. In Proc. FACS 2006, volume 182 of ENTCS, pages 107-122, 2007.

13. M. Kwiatkowska, G. Norman, and D. Parker. Game-based abstraction for Markov decision processes. In Proc. QEST'06, pages 157-166. IEEE CS Press, 2006.

14. M. Kwiatkowska, G. Norman, and D. Parker. Stochastic games for verication of probabilistic timed automata. Technical Report RR-09-05, Oxford University Computing Laboratory, 2009.

15. M. Kwiatkowska, G. Norman, D. Parker, and J. Sproston. Performance analysis of probabilistic timed automata using digital clocks. Formal Methods in System Design, 29:33-78, 2006.

16. M. Kwiatkowska, G. Norman, R. Segala, and J. Sproston. Automatic verification of real-time systems with discrete probability distributions. Theoretical Computer Science, 282:101-150, 2002.

17. M. Kwiatkowska, G. Norman, J. Sproston, and F. Wang. Symbolic model checking for probabilistic timed automata. Inf. and Comp., 205(7):1027-1077, 2007.

18. K. Larsen, P. Pettersson, and W. Yi. UPPAAL in a nutshell. International Journal on Software Tools for Technology Transfer, 1(1-2):134-152, 1997.

19. M. Puterman. Markov Decision Processes: Discrete Stochastic Dynamic Programming. John Wiley and Sons, 1994.

20. L. Shapley. Stochastic games. In Proc. National Academy of Science, volume 39, pages 1095-1100, 1953.

21. M. Sorea. Lazy approximation for dense real-time systems. In Proc. FORMATS/FTRTFT'04, volume 3253 of LNCS, pages 363-378. Springer, 2004.

22. S. Tripakis. The formal analysis of timed systems in practice. $\mathrm{PhD}$ thesis, Université Joseph Fourier, 1998.

23. S. Tripakis. Verifying progress in timed systems. In Proc. ARTS'99, volume 1601 of LNCS, pages 299-314. Springer, 1999.

24. S. Tripakis, S. Yovine, and A. Bouajjani. Checking timed Büchi automata emptiness efficiently. Formal Methods in System Design, 26(3):267-292, 2005. 